\title{
Scalable Online Learning in Physical Chemistry
}

\author{
Michael A. Nashªb
}

\begin{abstract}
The SARS-CoV-2/COVID-19 pandemic has disrupted higher education across the globe. As of early November 2020, Europe now finds itself in the middle of a second wave that is even more destructive than the first. The Swiss Federal Council declared on 28 October, 2020 that face-to-face teaching at Swiss Universities was to cease within days. With large introductory lectures in natural science faculties forced entirely online, educators in Switzerland are facing new challenges and dealing with the limitations of remote instruction. Through a series of anecdotes and observations, this article identifies challenges associated with scalable online learning, and explores methods to mitigate them. Additionally, several advantages to scalable online instruction are identified. By focusing on areas where online instruction has significant advantages, I argue that we can deliver high quality instruction in the chemical sciences remotely.
\end{abstract}

Keywords: Chemical education · COVID-19 - Online instruction · SARS-CoV-2

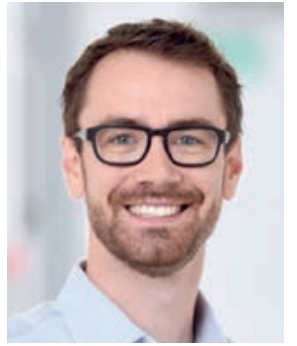

Prof. Michael Nash earned a Bachelor of Science in Biomedical Cybernetics from the University of California, Los Angeles in 2006 and a dual $\mathrm{PhD}$ in Bioengineering and Nanotechnology from the University of Washington, Seattle in 2010. Following Postdoctoral training in Applied Physics at Ludwig-Maximilians University in Munich, Nash began as tenure-track Assistant Professor with joint appointments at the University of Basel, Department of Chemistry and ETH Zurich, Department of Biosystems Science \& Engineering in 2016. Nash's research is in the area of molecular engineering and biophysics, specifically focusing on single-molecule protein biomechanics, protein engineering, and the interface between synthetic and biological systems.

\section{Background}

Introductory chemistry (German: Einführung in die Chemie) at the University of Basel is a large first semester chemistry lecture required for students in the faculty of natural sciences majoring in pharmaceutical science, biology, chemistry, computational science, geological science, and nanoscience. The course is worth six European Credit Transfer System (ECTS) points, and consists of 14 weeks of instruction in German. Each week of instruction is divided into three sessions: two lectures of two hours duration, and a recitation session of two hours duration organized and run by graduate student teaching assistants. In non-pandemic years, the lecture takes place in a large and spacious lecture hall on the University of Basel campus, located in the historic 'Bernoullianum'. Following the first introductory seven weeks taught by Prof. Oliver Wenger, I am responsible for teaching the second half of this course, covering aspects of physical chemistry, including states of matter, intermolecular interactions, solutions, colligative properties, mass action law, equilibrium, acids and bases, buffers, titration, thermodynamics, electrochemistry, and kinetics. The Fall Semester of 2020 will be the fifth time I have co-taught this course. The new challenges arising from remote teaching were felt by chemical educators across the globe, and several commentaries have already been published. ${ }^{[1-5]}$ This article adds commentary and anecdotal examples that are relevant for the situation in Switzerland.

\section{Non-pandemic Engagement}

Typically, there are just under 400 students enrolled in the course, with approximately half that number regularly attending the lectures. The size of the audience and their level of engagement in the material can contribute to a challenging didactic environment. How can such a large in-person lecture be optimally managed to maximize learning outcomes?

In a non-pandemic year, the format I have found to work well mixes traditional lectures on the chalk board with live audience polls. For the chalkboard lecture, I first introduce the concepts, explaining them from first principles, usually with the help of diagrams or molecular structures. After that, we work through an example problem that incorporates a set of given information and requires both conceptual understanding and accurate calculation to solve correctly. Following these activities, I test comprehension using a few poll-the-audience questions, where the students can vote on their mobile phones. The system I am working with is called 'PINGO' and is hosted by the University of Paderborn (pingo.upb.de). With this free online platform, I am able to solicit answers from the audience in real time, and display the results on the projector system.

For this introductory course, all the most classic physical and chemical experiences from everyday life serve as examples to connect the students with the material. Why do ice cubes float? Why does sweating cool you off? Why do we throw salt on the streets in the winter? Why does pasta take longer to cook at altitude? The questions themselves incorporate common knowledge of experience from daily life, but the answers serve as focal points for learning chemical concepts. I believe this connection to everyday experience can be engaging for the students. Many students are interested in learning about the practical applications of chemical concepts in the real world, and they appreciate these aspects being brought into the lecture. 


\section{Adjusting to Remote Teaching}

Due to the pandemic pressure, in the Fall Semester 2020 Einführung in die Chemie was offered as an online course only. All the lectures and exercises were moved to Zoom. The first challenges we faced were in adjusting to the new digital technology, and learning to navigate the platform. Many of my colleagues and I for the first time experienced curiosities and frustrations on these online platforms, and had to quickly learn to manage various video and audio settings, hosting rights, and system management in general.

In preparation for remote teaching, considering aspects such as lighting, camera setup, and the choice of microphone and tablet can go a long way. The best lighting is natural light. If you can face a window while teaching, the diffuse natural light will be superior to any artificial lighting generated from a desk lamp. Remove illumination sources from behind you, and make sure there are no shadows across your face in the video feed. When positioning my camera (iMac), I find that slightly above the level of the head works well, such that the camera slightly looks down from above. For audio, I find that a bluetooth headset with a boom microphone (Jabra) works best. Not only is the microphone close to my mouth, but my own voice is transmitted through the noise cancelling headphones. It allows me to gauge my volume effectively, and provides a huge improvement in sound quality compared to the internal microphone of my computer. Finally, I find that having a tablet (iPad) external from the CPU is essential, so that the call settings and chat window can be managed on the computer, and the slides can be shown by sharing the screen of the tablet. In practice, I have my slides as a PDF document that I load into a note taking app called Notability (Fig. 1). I can easily navigate through the PDF slide deck using the note taking app. In addition to extensive drawing and writing tools, this note taking app also has a laser pointer feature, allowing me to quickly switch between pointing to items on the PDF slides with the laser pointer, and drawing, writing over or highlighting the slides with the drawing tools. For me this hardware setup is reliable and working well.

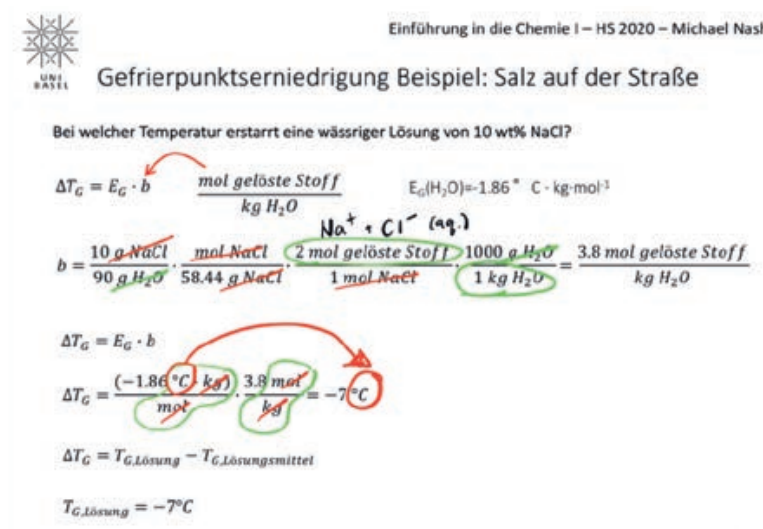

Fig. 1. Example of an annotated slide made in Notability during a German language online introductory chemistry lecture covering freezing point depression.

\section{300+ Zoom Lectures}

We opted for live synchronous online lectures, where all students are present on a Zoom call during the normal scheduled lecture period. Since we chose not to record the lectures, the online experience closely mimics that of the in-person lecture. Immediately, I noticed that attendance in the remote lecture sessions is much higher compared to normal in-person lectures. This year we regularly have $>350$ students logged in to the zoom call, whereas for in-person lectures $\sim 150$ students is more typical. Apparently, there are many students enrolled in our course will- ing to participate in remote instruction, but not willing to show up in person at the Bernoullianum. This suggests the possibility of increasing attendance in future non-pandemic years by offering a hybrid of in-person/remote instruction. In such a setup, students could attend the lecture in person, or have the option of following online remotely.

\section{New Aspects Enabled by Online Format}

I have found several new aspects are enabled by the online/ remote teaching platform that can positively impact the learning experience for the students. The most significant of these has been the live chat window available during the sessions. I encourage the students to type their questions into the chat window at any time during the lecture. After finishing a concept block, I then turn to the chat window and start reading some of the questions out loud, and providing the answers. I only check the chat window once I have finished a concept block, when I am at a good stopping point to take questions. What I found is that students started answering each others' questions in real time to compensate for the time delay. The ability for students to ask question in a more anonymous way, by typing into a chat window, really lowers the barrier for asking a question. In my experience, there is much higher level of question asking and participation. There is also a collaborative aspect to the lecture that is newly facilitated when students can interact directly with each other in the chat window, ask questions, and provide answers to their peers. With the new online format, I estimate I receive approximately four times the number of questions from the audience. Although this can slow down the pace with which we can cover the material, the nature of these questions serves as an indicator to me as the instructor which concepts are problematic and need more reinforcement, and which ones are well understood. Also the lecture becomes more engaging for those students choosing to participate in the live chat.

These two strong points in remote teaching, i.e. massively increased attendance and massively increased question/answer sessions facilitated through the live chat window, are significant improvements enabled by the online platform. The opportunity for the stronger students to directly answer in real time the questions from the weaker students facilitates peer-to-peer teaching and learning. I believe this aspect should not be underestimated, and could significantly improve learning and comprehension for these students.

\section{Professionalism in Digital Environments}

At the start of the course, I made a few comments about behaving responsibly in the digital environment. The one issue we had was when one student told another classmate to stop asking so many questions in the chat window. It escalated somewhat and the students exchanged some bitter words in the chat window. Also, I made the mistake of inviting the audience to turn on their cameras during one of my first online lectures. I thought if we could all see each other, it would facilitate a sense of community in the course, and allow us to interact more easily. The first consequence was that the bandwidth consumption was too much with $>350$ people logged in. This led to glitches and delays in the video feed. Also, not everyone in the audience was prepared to look professional. For example, some students wore their pajamas or were seen smoking during the lecture. For these large synchronous courses, it works better if the audience has their cameras switched off.

\section{Outlook}

We don't know how long remote learning will be required in Swiss higher education. We all hope that by 2022 our courses could potentially be back to normal, however, there remain huge uncertainties with regards to the status of the pandemic on 
timescales of only a couple weeks. I believe, however, that this rapid forceful transition to remote learning will have a lasting effect. It raises fundamental questions about the mission of the University and the role of instructors when the students are no longer required to be physically present to earn their degrees. The increased attendance and increased audience participation facilitated by the live chat window in synchronous online lectures that I have witnessed as part of the introductory chemistry course is certainly a positive signal that online instruction is a competitive medium. When considering other outside factors, including aspects such as student safety, COVID-19 measure compliance, scalability, and accessibility, it would not be surprising to see remote instruction for some lectures at Swiss cantonal universities and federal institutes of technology to be adopted on the long term. By focusing on these positive aspects of remote online instruction, I believe we as chemical educators can provide high quality learning environments and effectively prepare the student cohorts for their future educational and professional development, whatever direction they choose to take.

\section{Acknowledgements}

I would like to thank Prof. Oliver Wenger at the University of Basel for leading the introductory chemistry course, and handling all logistical and administrative aspects over the last 5 years.

Received: November 18, 2020
[1] K. Wilson, J. Chem. Educ. 2020, 97, 2895, https://doi.org/10.1021/acs.jchemed.0c00702

[2] J. M. Nyachwaya, J. Chem. Educ. 2020, 97, 2935, https://doi.org/10.1021/acs.jchemed.0c00891

[3] I. P. O'Carroll, M. R. Buck, D. P. Durkin, W. S. Farrell, J. Chem. Educ. 2020, 97, 2383, https://doi.org/10.1021/acs.jchemed.0c00710

[4] J. Rodríguez Núñez, J. Leeuwner, J. Chem. Educ. 2020, 97, 2819, https://doi.org/10.1021/acs.jchemed.0c00781

[5] S. S. Schweiker, S. M. Levonis, J. Chem. Educ. 2020, 97, 2863, https://doi.org/10.1021/acs.jchemed.0c00621

\section{License and Terms}

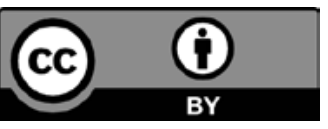

This is an Open Access article under the terms of the Creative Commons Attribution License CC BY 4.0. The material may not be used for commercial purposes.

The license is subject to the CHIMIA terms and conditions: (http:// chimia.ch/component/sppagebuilder/?view=page \&id=12).

The definitive version of this article is the electronic one that can be found at https://doi.org/10.2533/chimia.2021.64 